\title{
Penfigoides: \\ Revisión y puesta al día
}

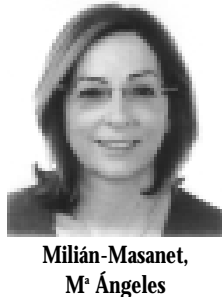

Pemphigoid: Revision and update

Milián- Masanet, Ma Angeles* Sanchis- Bielsa, José $M^{* *}$

\footnotetext{
* Profesora Asociada de Medicina Bucal. Universidad de Valencia.

** Profesor Asociado de Cirugía Oral y Médico Adjunto del Consorcio Hospital General Universitario de Valencia.
}

Resumen: Las enfermedades vesículo-ampollares de la mucosa oral pueden estar producidas por agentes mecánicos, físicos o químicos, por microorganismos, o por desórdenes inmunológicos o alérgicos. Dentro de los procesos ampollares de base inmunológica se encuentran el eritema exudativo multiforme, el pénfigo vulgar, el penfigoide, el liquen plano, el lupus eritematoso, la dermatitis herpetiforme y la enfermedad por depósito lineal de IgA. Este artículo presenta una revisión de los diferentes aspectos etiopatogénicos, clínicos, y terapéuticos de los penfigoides dentro de las enfermedades ampollares de la cavidad oral. Además se realiza una puesta al día, exponiendo los criterios diagnósticos, así como las técnicas más importantes empleadas en el mismo.

Palabras clave: Penfigoide, Mucosa oral, Enfermedad ampollar.

Abstract: Disorders characterized by vesicle-blister lesions of the oral mucosa can be caused by mechanical, physical or chemical agents, microorganisms, or immune or allergic alterations. The ampullar processes of immune origin include exudative erythema multiforme, pemphigus vulgaris, pemphigoid, lichen planus, lupus erythematosus, herpetiform dermatitis and linear IgA disease.

A review is provided of the different etiopathogenic, clinical and therapeutic aspects of pemphigoid disorders among the range of ampullar diseases of the oral cavity. An update is also provided, with a description of the diagnostic criteria and principal techniques employed.

Key words: Pemphigoid, Oral mucosa, Ampullar diseases.
Mạ Angeles Milián Masanet

C/ San Vicente Mártir 102, 13a

46007-Valencia

E-mail: claves@wanadoo.es
BIBUD [1138-123X (2004)9:4; julio-agosto 361-476]

Milián-Masanet MA, Sanchis-Bielsa J M. Penfigoides: revisión y puerta al día. RCOE 2004;9(3):429-434. 


\section{Introducción}

El término penfigoides describe a un grupo de enfermedades inflamatorias crónicas, de naturaleza autoinmune, que afectan a la piel o a las membranas mucosas, rara vez afecta a ambas. Cuando se producen lesiones cutáneas no suele haber cicatrices como secuela y al proceso se le denomina penfigoide bulloso o ampollar $(\mathrm{PB})$, mientras que si el proceso se localiza en las mucosa con frecuencia se producen cicatrices y se denomina penfigoide de las membranas mucosas $(\mathrm{PMM})^{1}$, aunque a veces se pueden observar lesiones en la piel. Las mucosas más frecuentemente afectadas suelen ser la oral seguida de la ocular; también se pueden producir lesiones en el esófago, nasofaringe y laringe. Otras denominaciones de este proceso son penfigoide cicatrizal, penfigoide benigno de las membranas mucosas, pero puesto que algunas lesiones no producen cicatrices y en algunos casos el proceso no tiene un curso tan benigno (con importantes secuelas) estos nombres se prefiere no utilizarlos. Puesto que en el PB las lesiones son fundamentalmente cutáneas, y las lesiones orales cuando se producen son idénticas a las del PMM, nos referiremos en este trabajo al PMM.

El PMM se define pues como un grupo de enfermedades autoinmunes, inflamatorias crónicas, con formación de ampollas subepiteliales, que predominantemente afecta a las membranas mucosas y que se caracteriza por un depósito lineal de lgG, IgA o C3 a lo largo de la membrana basal ${ }^{2+*}$.

Clínicamente se manifiesta como ampollas bien intactas o erosiones debi- do a la rotura de las ampollas. En la cavidad oral las lesiones se localizan sobre todo en las encías y el paladar, apareciendo en la primera como un eritema intenso de la encía insertada, que se denomina gingivitis descamativa crónica (GDC). La GDC no es exclusiva del PMM, ya que aparece en otros procesos como el liquen plano y el pénfigo vulgar, con los que habrá que plantear un diagnóstico diferencial en base a la clínica y al estudio histopatológico.

El interés del conocimiento de esta entidad radica en que el diagnóstico precoz y por lo tanto su tratamiento y control mejora el pronóstico de la enfermedad, ya que se ha observado que a más tiempo desde el diagnóstico hasta el tratamiento, mayor tiempo de tratamiento se requiere ${ }^{3}$. Por otra parte también es importante que el dentista tenga en cuenta este proceso ya que debido a la frecuente localización de las lesiones en la cavidad oral será el primero en observarlas y en el caso de la GDC hay que evitar el confundirlas con otros procesos gingivales o periodontales.

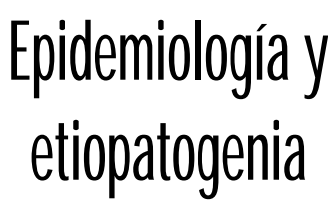

El PMM es una enfermedad propia de la edad adulta, con una edad media de 60 años, la mayoría de los casos entre los 40 y los 80 años. Es más frecuente en mujeres en una proporción de dos a uno con respecto a los hombres'. No hay predilección racial o geográfica. Con respecto al PB es mucho menos frecuente, con una relación de siete a uno, sin embargo como las referencias están tomadas de datos dermatológicos quizás de ahí la mayor proporción del $\mathrm{PB}^{5}$.

Con relación a otros procesos autoinmunes el penfigoide es más frecuente que el pénfigo vulgar (3:1), pero menos que el liquen plano (1:15).

Su etiología es desconocida hoy en día, pero se sabe que es un proceso de naturaleza autoinmune en el que se producen autoanticuerpos contra componentes de la membrana basal del epitelio, en concreto contra la lámina lúcida. Se han detectado 10 diferentes componentes de la zona de la membrana basal por medio de autoanticuerpos de pacientes con PMM, unos con identidad conocida y otros desconocida. De ellos los más importantes son los antígenos del PB 1 y 2 , laminina 5 , laminina 6 , colágeno tipo VII y la subunidad beta 4 de la integrina. Sin embargo hasta el momento actual no hay evidencia que apoye una correlación entre los diferentes subtipos clínicos y autoanticuerpos específicos ${ }^{2 *}$. Ultraestructuralmente se ha observado que los autoanticuerpos del PMM reaccionan con epitopes de la zona del antígeno del PB 2 pero son diferentes de los epitopes que reaccionan con el $\mathrm{PB}^{6}$.

Por otra parte se ha observado una base inmunogenética, ya que se ha encontrado una asociación con el HLA DQB1*03017. Las moléculas HLA-DQ en la superficie de las células presentadoras de antígeno funcionan inmunológicamente presentando el antígeno a las células $\mathrm{T}$. Posiblemente también existe un papel de la inmunidad celular y de las citoquinas en la patogénesis del $\mathrm{PMM}^{2}$.

Recientes investigaciones refieren que una inflamación crónica puede

RCOE, 2004, Vol 9, №4, 429-434 
ser responsable del desencadenamiento del PMM por un fenómeno inmune llamado «extensión del epitope», el cual se refiere a que una enfermedad crónica o autoinmune produce un daño tisular en el que ciertos antígenos ocultos de las células T o B autorreactivas quedan mostrados $\mathrm{y}$ provocan una enfermedad autoinmune secundaria o primaria respectivamente ${ }^{2 * *}$.

\section{Cuadro clínico}

Las mucosa oral, ocular y genital son las más frecuentemente afectadas, seguidas en orden de frecuencia por la faríngea, laríngea, nasal y esofágica. Se producen ampollas que se rompen dando lugar a erosiones que pueden producir o no cicatrices.

En la cavidad oral la localización más frecuente es la encía y el paladar ${ }^{8}$, siendo característica la manifestación clínica denominada gingivitis descamativa crónica (GDC) ${ }^{9,10}$ en la que la encía insertada aparece eritematosa, brillante y a veces hemorrágica, produciendo molestias e incluso dolor, debido a la ruptura de las ampollas, que se produce en horas, con una curación habitualmente sin cicatrices. Las ampollas por lo tanto se aprecian ocasionalmente, aunque lo que se puede observar es el techo de la misma que en ocasiones se conserva a pesar de la ruptura (figs. 1-6). Existe signo de Nikolsky positivo ${ }^{11}$, provocándose la formación de una ampolla con la presión del dedo, con el espejo dental o con una sonda periodontal ${ }^{1}$. Las lesiones pueden aparecer repetidamente en el mismo sitio y el desencadenamiento puede estar provocado por el trauma de la masticación o bien la presión de una prótesis removible.

La GDC no aparece exclusivamente en el PMM, ya que otros procesos como el liquen plano ( $P$ ) y el pénfigo vulgar (PV) también pueden mostrar afectación gingival, así como la dermatitis herpetiforme, la enfermedad por depósito lineal IgA, el eritema multiforme, o la epidermolisis bullosa. En ellos el estudio clínico exhaustivo unido al estudio histopatológico y de inmunofluorescencia directa son fundamentales para concretar de que enfermedad se trata. Así en el LP buscaremos la presencia de las características estrías blanquecinas en mucosas yugales, mientras que en el PV es más difícil la diferenciación clínica, ya que al inicio las lesiones suelen estar más localizadas y las ampollas también se rompen dando lugar a erosiones, quizás algo menos hemorrágicas debido a que la ampolla es intraepitelial. En el PV la biopsia será de gran importancia para poder planificar el tratamiento mucho más potente que el del PMM.

La afectación ocular es importante en el PMM ya que las lesiones suelen estar presentes en el $85 \%$ de los casos. Al inicio los pacientes refieren conjuntivitis, que después progresa a ulceración, con formación de bridas cicatriciales, entropión e incluso ceguera por afectación corneal severa y simbléfaron. La fibrosis y cicatrices pueden producir sequedad ocular debido a la obliteración del conducto lacri$\mathrm{mal}^{4}$. De ahí la importancia de preguntar al paciente en la anamnesis acerca de molestias oculares, de explorarlo para descartar la presencia de bridas cicatriciales y de remitirlo al oftalmólogo si tenemos la confirmación histo- lógica de PMM para descartar o confirmar lesiones oculares.

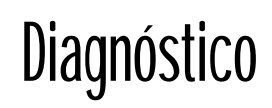

Además de las características de las lesiones clínicas, que se incluyen en los criterios diagnósticos según The First International Consensus ${ }^{2 * *}$, el diagnóstico se basa en los resultados de los estudios histológicos e inmunopatológicos ${ }^{12 * *}$.

Histología: con tinción con hematoxilina-eosina se observa una ampolla subepitelial en la que el epitelio queda despegado del conectivo a nivel de la membrana basal. Se puede observar asociado un infiltrado inflamatorio en las áreas papilares del tejido conectivo $^{13^{* *}}$, así como leucocitos polimorfonucleares en el interior de la vesículaampolla.

Otros procesos cursan también con presencia de ampollas subepiteliales, como el $L$, el lupus eritematoso, la epidermolisis bullosa adquirida, la enfermedad por depósito lineal de $\lg \mathrm{A}$, y es el estudio inmunopatológico el que llevará al diagnóstico definitivo.

Es importante la técnica de biopsia, ya que si no es correcta el diagnóstico será inespecífico y se tendrá que volver a repetir la toma de una muestra. Para ello habrá que realizarla en una zona que abarque parte de lesión así como tejido sano próximo a la lesión, donde se pueden observar el inicio de la formación de la ampolla ${ }^{14}$. Inmunofluorescencia directa (IFD): se observa una distribución uniforme en forma de banda o línea a nivel de la membrana basal, positiva para lgG y C3 sobre todo ${ }^{15,16}$; a veces también hay positividad para la $\lg \mathrm{A} o \lg \mathrm{M}^{5}$. 


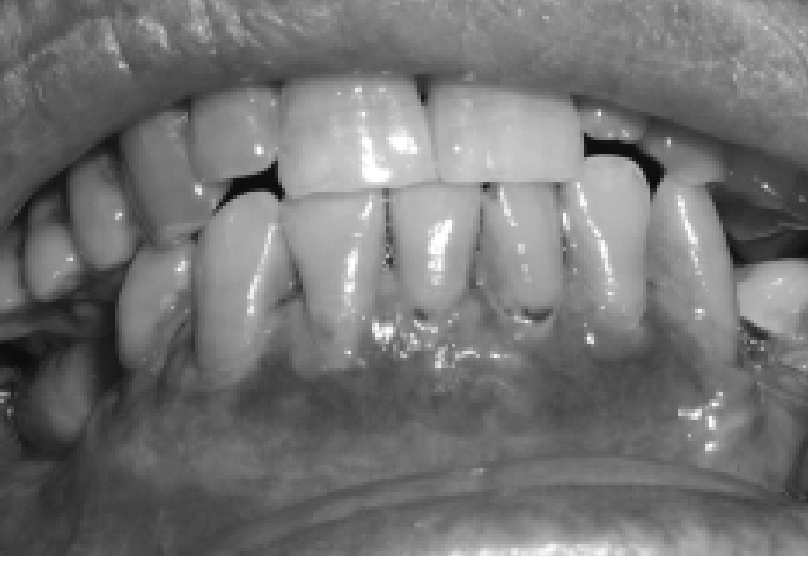

Figura 1. GDC en la arcada inferior. Se puede observar la afectación de la encía insertada.

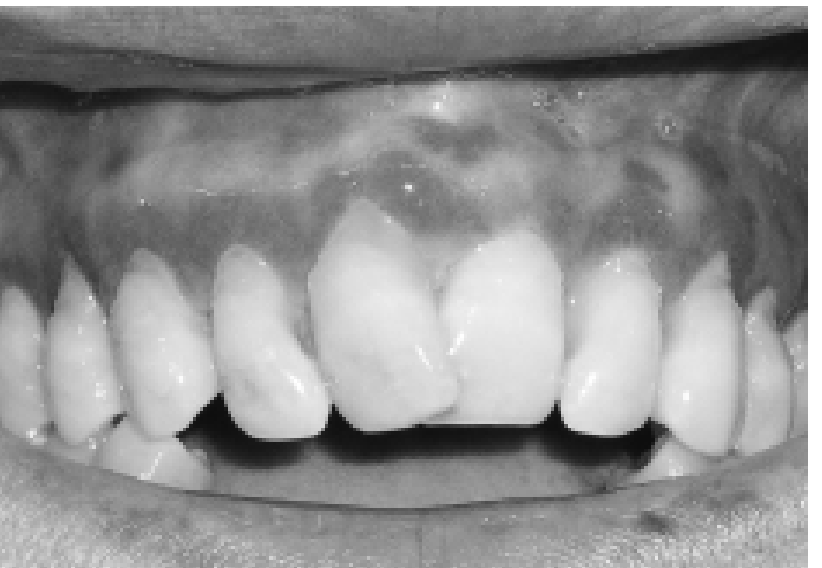

Figura 3. GDC localizada en la encía superior.

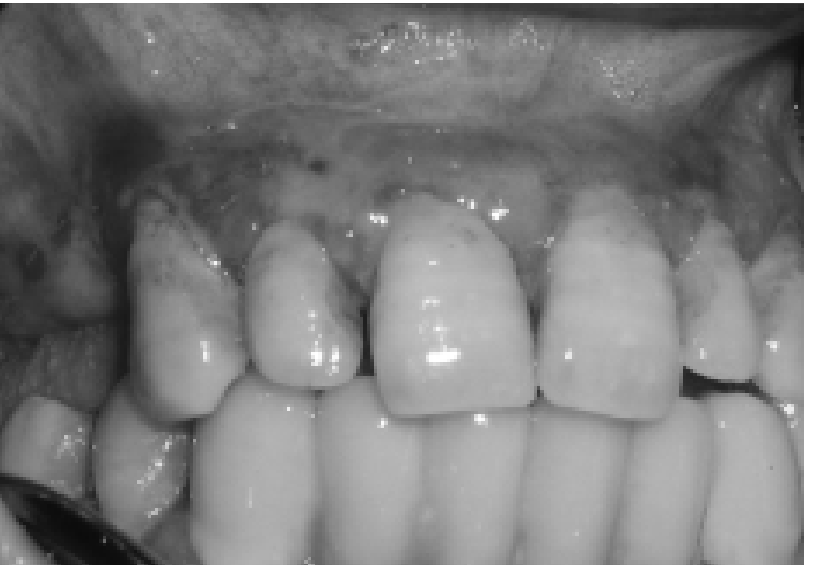

Figura 5. Detalle a mayor aumento de la figura anterior.

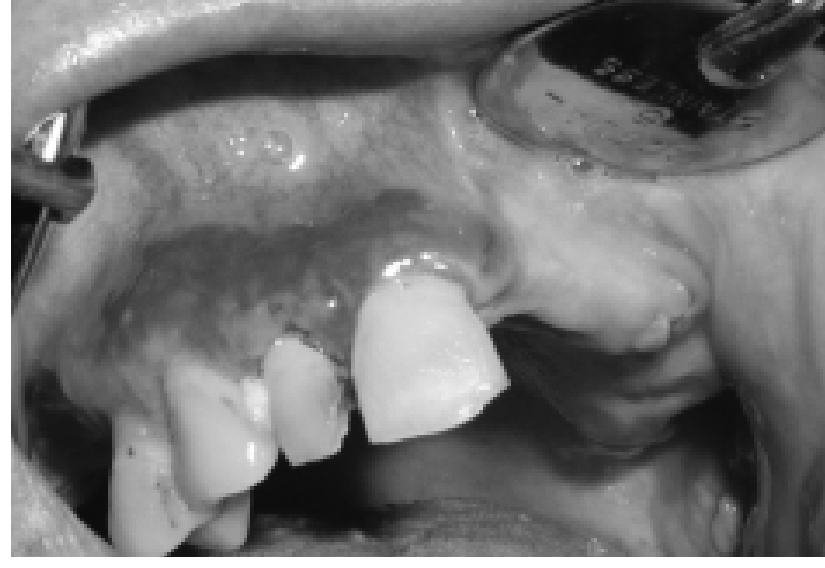

Figura 2. GDC en la arcada superior.

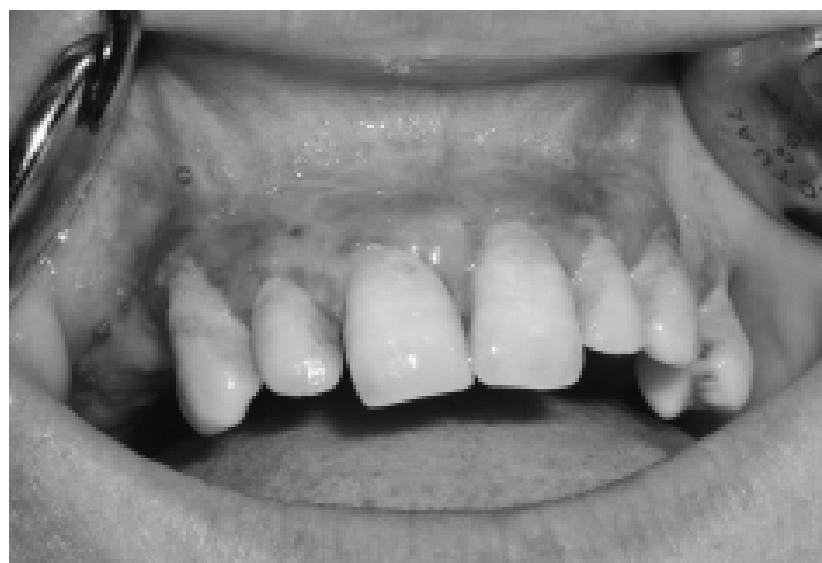

Figura 4. Lesiones de penfigoide en la encía superior. Obsérvese la presencia de erosiones en diferentes zonas de la misma.

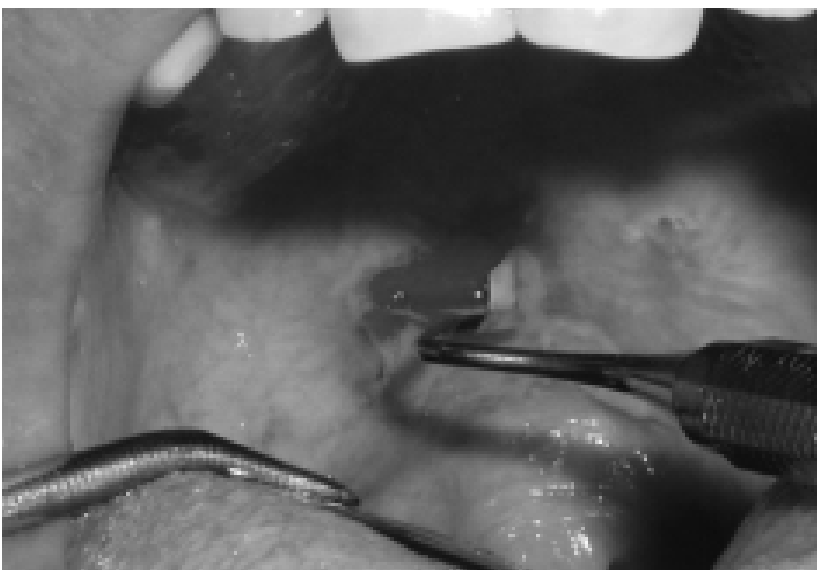

Figura 6. Ampolla rota en el paladar blando, en la que permanece el techo de la misma.

RCOE, 2004, Vol 9, №4, 429-434 
Inmunofluorescencia indirecta (IFI): se utiliza para detectar anticuerpos circulantes en el suero del paciente. No es una técnica muy utilizada ya que no siempre resulta positiva en los pacientes con PMM y además las titulaciones no se han confirmado que se correlacionen con el curso de la enfermedad en relación a la severidad y actividad. Según The First International Consensus es recomendable la realización de esta técnica, pero no es criterio absoluto de diagnóstico ${ }^{2 * *}$. Su utilidad radica en la detección de los diferentes autoantígenos detectados por los autoanticuerpos de los pacientes. Mediante técnicas complejas como la IFI sobre piel dividida con cloruro sódico, se aumenta la sensibilidad de la IFI, y permite la distinción entre aquellos antígenos que están localizados en el lado epitelial (zona superior de la lámina lúcida) de aquellos localizados en el lado dérmico (zona inferior de la lámina lúcida) ${ }^{13}$. Sin embargo, técnicas más sofisticadas como la inmunoprecipitación que permiten diferenciar los antígenos diana y por lo tanto el proceso concreto no se realizan en todos los centros hospitalarios.

\section{Tratamiento}

En el paciente con PMM hay que tener en cuenta diferentes factores a la hora de planificar el tratamiento. Por una parte si el paciente ha debutado con lesiones orales, que suele ser lo más frecuente, hay que excluir lesiones en otras mucosas para lo cual se remitirá a los diferentes especialistas: ginecólogo, oftalmólogo, otorrinolaringólogo, puesto que el tratamiento será diferente si las lesiones están localizadas sólo en la mucosa oral o si hay más localizaciones y además es importante para evitar que no se produzcan secuelas como ceguera, obstrucción aérea, o disfunciones sexuales o urinarias.

Por otra parte además de la localización hay que tener en cuenta la severidad de las lesiones así como la rapidez de la extensión de las mismas, que obligarán a pautar una medicación más potente. Según The First International Consensus ${ }^{2 * *}$ los pacientes se pueden clasificar en dos grupos según los factores mencionados:

A. Alto riesgo (múltiples localizaciones): se administra corticoides por vía oral, prednisona a dosis de 40-60 $\mathrm{mg} /$ día $01 \mathrm{mg} / \mathrm{Kg} /$ día, asociado a ciclofosfamida (1-2 mg/Kg/día), o azatioprina (1-2 mg/Kg/día). Otra vía alternativa es la dapsona (fármaco del grupo de las sulfonas)(50-200mg/día) ${ }^{3}$. Este último se pauta incrementando la dosis progresivamente, primero 25 mg/día y cada 3 días se aumenta 25 mg hasta los 100 mg/día; posteriormente se sube la dosis cada semana hasta el máximo de 200 mg. Además antes de su administración es importante descartar deficiencia de la glucosa-6-fosfato deshidrogenasa ${ }^{17}$ para evitar efectos secundarios importantes.
B. Bajo riesgo (sólo mucosa oral): Corticosteroides tópicos de potencia moderada o alta, aplicados en forma de pomada o colutorios $^{18}$ en casos de lesiones no muy extensas. Así el propionato de clobetasol al 0,05\%o el acetónido de fluocinolona al $0,05 \%$ suele funcionar satisfactoriamente, y en ocasiones se pueden colocar en férulas hechas a medida de la arcada del paciente que facilita la aplicación del fármaco ${ }^{19 *}$. Si no se obtiene respuesta clínica se pauta dapsona a la dosis anteriormente comentada. En los casos con lesiones extensas intraorales es necesario administrar corticoides a bajas dosis (prednisona 0,5 mg/Kg/día).

Otros tratamientos referidos recientemente con resultados satisfactorios en casos aislados son la talidomida (100 mg/día) $)^{20}$, el etanercept (25 $\mathrm{mg} / 2$ veces a la semana/subcutáneamente) $)^{21}$, o la asociación de nicotinamida y tetraciclina ${ }^{22}$.

En relación al pronóstico de los pacientes, se ha observado que depende de los lugares afectados. Así la localización en la mucosa oral sola o asociada a la piel se asocia a mejor pronóstico. Asimismo la presencia de autoanticuerpos IgG e IgA contra la membrana basal se relaciona con mayor severidad y persistencia de la enfermedad, y los títulos de anticuerpos cuando se detectan podrían estar relacionados con la actividad de la misma. Sin embargo no parece que haya relación entre los autoanticuerpos antígeno-específicos y el pronóstico de la enfermedad ${ }^{2 * *}$. 


\section{Bibliografía recomendada}

Para profundizar en la lectura de este tema, el/los autor/es considera/an interesantes los artículos que aparecen señalados del siguiente modo: *de interés $* *$ de especial interés.

1. Dayan S, Simmons RK, Ahmed AR. Contemporary issues in the diagnosis of oral pemphigoid. A selective review of the literature. Oral Surg Oral Med Oral Pathol Oral Radiol Endod 1999;88:424-30.

2.** Chan LS, Ahmed R, Anhalt GJ, Bernauer W, Cooper KD, Elder MJ, et al. The First International Consensus on Mucous Membrane Pemphigoid. Arch Dermatol 2002;138:370-9. Este artículo es de especial interés porque en él 26 participantes aunaron criterios sobre los diferentes aspectos etiopatogénicos, clínicos, diagnósticos y terapéuticos del PMM.

3. Mobini N, Nagarwalla N, Ahmed R. Oral Pemphigoid. Subset of cicatricial pemphigoid? Oral Surg Oral Med Oral Pathol Oral Radiol Endod 1998;85:37-43.

4. Williams DM. Vesiculo-bullous mucocutaneous disease:benign mucous membrane and bullous pemphigoid. J Oral Pathol Med 1990;19:16-23.

5. Scully C, Carrozzo M, Gandolfo S, Puitatti P, Monteil R. Update on mucous membrane pemphigoid. Oral Surg Oral Med Oral Pathol Oral Radiol Endod 1999;88:56-68.

6. Parisi E, Raghavendra S, Werth VP, Sollecito TP. Modification to the approach of the diagnosis of mucous membrane pemphigoid: a case report and literature review. Oral Surg Oral Med Oral Pathol Oral Radiol Endod 2003;95: 182-6.

7. Yunis JJ, Mobini N, Yunis EJ, Alper CA, Deulofeut R, RodriguezA, et al. Common major histocompability complex class II markers in clinical variants of cicatricial pemphigoid. Proc Natl Acad Sci 1994;91:7747-51.

8. Bagán JV, Peñarrocha M, Milián MA. Pénfigo vulgar $y$ penfigoide benigno de la mucosa:estudio de sus manifestaciones orales en $\mathbf{2 4}$ casos. Av Odontoestomatol 1993;9:387-92.

9. Bozkurt FY, Celenligil H, Sungur A, Huracán S. Gingival involvement in mucous membrane pemphigoid. Quintessence Int 1998;29:438-41.

10. Castellanos Suarez JL. Enfermedades gingivales de origen inmune. Med Oral 2002;7:271-83.

11. Terezhalmy GT, Bergfeld WF. Cicatricial pemphigoid (benign mucous membrane pemphigoid). Quintessence Int 1998;29:429-37.

12. Bermejo A, López P. Diagnóstico de las enfermedades vesiculares y ampollares de la mucosa bucal: desórdenes de la cohesión intraepitelial y de la unión epitelio-conectiva. Med Oral 1996;1:23-43.

13**. Hernández Vallejo G, Arriba de la Fuente L, Lucas Tomás M. Enfermedades ampollosas de la mucosa bucal. Características diferenciales clínicas, histológicas e inmunológicas. Med Oral 1999;4:528-51.

En este artículo de revisión el autor realiza un diagnóstico diferencial de las enfermedades ampollares de la mucosa oral de naturaleza autoinmune, en relación a los diferentes aspectos clínicos, histopatológicos, e inmunopatológicos.

14. Weinberg MA, Insler MS, Campen RB. Mucocutaneous features of autoimmune blistering diseases. Oral Surg Oral Med Oral Radiol Endod 1997;84:517-34

15. Vincent SD, Lilly GE, Baker KA. Clinical, historic, and therapeutic features of cicatricial pemphigoid. A literature review and open therapeutic trial with corticosteroids. Oral Surg Oral Med Oral Pathol 1993;76:453-9.

16. Helander SD, Rogers RS. The sensitivity and specificity of direct immunofluorescence tes- ting in disorders of mucous membranes. $\mathrm{J}$ Am Acad Dermatol 1994;30:65-75.

17. Huilgol SC, Black MM. Management of the immunobullous disorders.I. Pemphigoid. Clinical and Experimental Dermatology 1995;20:189-201.

18. Gonzalez-Moles MA, Morales P, RodriguezArchilla A, Ruiz-Avila I, Gonzalez-Moles S. Treatment of severe chronic oral erosive lesions with clobetasol propionate in aqueous solution. Oral Surg Oral Med Oral Pathol Oral Radiol Endod 2002;93:264-70.

19*. Gonzalez-Moles MA, Ruiz-Avila I, RodriguezArchilla A y cols. Treatment of severe erosive gingival lesions by topical application of clobetasol propionate in custom trays. Oral Surg Oral Med Oral Pathol Oral Radiol Endod 2003;95:688-92.

En este artículo se realiza un estudio sobre 33 pacientes con lesiones gingivales erosivas (22 con PMM y 11 con liquen plano oral) tratados con cubetas de acrílico para la aplicación de una pomada de propionato de clobetasol al 0,05\% y se exponen los resultados satisfactorios.

20. Duong DJ, Moxley RT, Kellman RM, Pincus SH, Gaspari AA. Thalidomide therapy for cicatricial pemphigoid. J Am Acad Dermatol 2002;47:193-5.

21. Sacher C, Rubbert A, Konig C, ScharffetterKochanek K, Krieg T, Hunzelmann N. Treatment of recalcitrant pemphigoid with tumor necrosis factor alfa antagonist etanercept. $J$ Am Acad Dermatol 2002;46:113-5.

22. Mallon E, Wojnarowska F. Cicatricial pemphigoid presenting with unusual palmar involvement, successfully treated with a combination of nicotinamide and tetracycline. Clinical and Experimental Dermatology 1994;19:526-30.

RCOE, 2004, Vol 9, №4, 429-434 